the AIDS epidemic, KS incidence in Bulawayo was similar to that in Kyadondo in both genders; however, in men it became higher in Harare than Kyadondo during the AIDS epidemic, thus seeming to follow the geographical distribution of HIV prevalence. In women, the geographical pattern of KS appeared independent of HIV prevalence. In the populations studied, despite HIV prevalence being higher in females than males, KS incidence was higher in males than females.

Conclusion HIV prevalence could have different impacts on KS incidence, possibly explained by other risk factor exposures. Furthermore, a higher risk of KS emergence in men or increased risk factor exposure in men could explain the gender differences.

\section{SP3-68 A SYSTEMATIC REVIEW OF INTERVENTIONS TO PREVENT OR REDUCE SUBSTANCE USE AND SEXUAL RISK BEHAVIOUR IN YOUNG PEOPLE}

doi:10.1136/jech.2011.1429760.68

C Jackson, ${ }^{*}$ R Geddes, S Haw, J Frank. Scottish Collaboration for Public Health Research and Policy, Edinburgh, UK

Background Interventions aimed at preventing risky behaviour in adolescence and young adulthood have largely focused on single risk behaviours and a limited number of underlying predictors. Interventions that take a broader approach to reducing risk behaviour may more effectively and efficiently reduce multiple risk behaviours. Methods We performed a systematic review to identify experimental studies of interventions to reduce risk behaviour in adolescents or young adults and that reported on any substance (alcohol, tobacco and illicit drug) use and sexual risk behaviour outcomes.

Results From 1129 articles, 18 experimental studies met our inclusion criteria, which were heterogeneous in nature and design. Study results were mixed, with programmes generally impacting on some outcome measures, but not others. The most promising interventions addressed multiple domains (individual and peer, family, school and community) of risk and protective factors for risk behaviour. There was some evidence that intervening in the early- to mid childhood school years has a high impact on later risk behaviour. Conclusions We found few studies that demonstrated a consistent, significant and sustained impact on risk behaviours. However, there is some evidence that programmes can have a long-term impact on multiple risk behaviours, with the most promising interventions addressing multiple domains of risk and protective factors. Complex interventions therefore need to be developed in the context of effective cross-sector engagement and collaboration. Furthermore, the current focus on investing in pre-school "early years" programmes should be extended to include the early- to mid-childhood school years.

\section{SP3-69 GENDER, ETHNIC AND FOOD INTAKE DIFFERENCES IN THE OCCURRENCE OF OVERWEIGHT IN INDIAN, NEPALESE AND SRILANKAN MEDICAL STUDENTS}

doi:10.1136/jech.2011.1429760.69

${ }^{1} \mathrm{~B}$ Sathian, ${ }^{*} \mathrm{R}$ Babu, ${ }^{2} \mathrm{~J}$ Sreedharan, ${ }^{1} \mathrm{~N}$ Bhat, ${ }^{1} \mathrm{~N}$ Chandrasekharan, ${ }^{3} \mathrm{E}$ Rajesh. ${ }^{1}$ Manipal College of Medical Sciences, Pokhara, Nepal; ${ }^{2}$ Gulf Medical University, Ajman, United Arab Emirates; ${ }^{3}$ MG University, Kerala, India

Introduction In developed and developing countries young people are at increased risk of obesity. Obesity is associated with chronic disease including Type II Diabetes, cardiovascular diseases and cancer. Body mass index (BMI) is commonly used to measure obesity. The aim of this study was to describe the prevalence of overweight among medical students in Nepal according to sex, ethnicity and diet (vegetarian/non-vegetarian).
Methods Approval for the study was obtained from the institutional research ethical committee. Participants were recruited from Manipal College of Medical Sciences, Pokhara, Nepal. BMI $\left(\mathrm{kg} / \mathrm{m}^{2}\right)$ was calculated using the height and weight. Data were analysed using Microsoft Excel (Windows 2003) and SPSS 16.

Results Of the 311 participants 179 (57.6\%) were Nepalese, 97 (31.2\%) Indian and 35 (11.2\%) Srilankan. $72 \%$ were males and $28 \%$ females. $80.1 \%$ were non-vegetarians and $19.9 \%$ were vegetarians. Overall $28.7 \%$ of subjects were overweight; $33 \%$ of Nepalese participants, $17.5 \%$ of Indian participants and $20 \%$ of Srilankans participants. $34.4 \%$ of men and $6.9 \%$ of women were overweight. $26.9 \%$ of non-vegetarian and $25.8 \%$ of vegetarian participants were overweight.

Conclusion In medical students in Nepal, gender, ethnicity and diet were associated with being overweight. Obesity prevention should focus on physical activity and diet.

\section{SP3-70 A NEW MODEL FOR ENVIRONMENTAL HEALTH DECISION MAKING AT A LOCAL LEVEL}

doi:10.1136/jech.2011.1429760.70

R Khan, ${ }^{*}$ D Lemon, D Phillips, E Youngman, R Partridge. NHS Dorset, Dorchester, Dorset, UK

Most environmental health issues are multi causal with complex non-linear relationships between the environment and health. New tools and methods are needed to assist policy and decision making that cut across traditional policy making structures and support collaboration by different agencies at different administrative and spatial scales. This project is aimed at developing a new environmental health decision making model to support integrated policy and action in environmental health, especially at a local level. We are piloting the model using both a setting based approach (Housing domain) and a health outcome approach (Chronic Obstructive Pulmonary disorder). The proposed decision making model is based on four stages: problem framing, environmental health intelligence, model building and appraisal. The problem framing stage involves conceptualising the problem using an ecological framework. The second stage involves developing environmental health indicators. The third stage involves converting the conceptual model into a simulation model using a system of differential equations and systems dynamics modelling. Appraisal represents the final stage in assessment, and provides the point at which results are synthesised and interpreted by the stakeholders. Results of the model will be presented at the conference. The aim of the decision making model is to enable stakeholders to understand the dynamics of environmental health issues and simulate and compare and evaluate different policy decisions and their financial and health impacts. This project will improve on current methods and tools to assess policy relevant problems that have implications for working across the environment and human health interface.

\section{SP3-71 QUALITY ASSESSMENT OF DIRECTLY OBSERVED TREATMENT OF TUBERCULOSIS IN DHAKA CITY}

doi:10.1136/jech.2011.142976o.71

${ }^{1} \mathrm{~S}$ Satter, ${ }^{*}{ }^{2} \mathrm{M}$ A Hafez, ${ }^{3} \mathrm{M}$ Flora, ${ }^{2} \mathrm{M}$ Shahjahan, ${ }^{2} \mathrm{~A}$ Afroz. ${ }^{1}$ International Center for Diarrhoeal Disease Research, Bangladesh (ICDDR,B), Dhaka, Bangladesh; ${ }^{2}$ Bangladesh Institute of Health Sciences (BIHS), Dhaka, Bangladesh; ${ }^{3}$ National Institute of Public Health \& Social Medicine (NIPSOM), Dhaka, Bangladesh

Introduction Quality of directly observed treatment (DOT) is key factors for successful TB control programme. This study was 